\title{
Mask-Assisted Seeded Growth of Segmented Metallic Heteronanostructures
}

\author{
Cameron C. Crane,$^{\dagger}$ Jing Tao ${ }^{\ddagger}$ Feng Wang, ${ }^{\dagger}$ Yimei Zhu ${ }^{\ddagger}$ Jingyi Chen ${ }^{\dagger, *}$ \\ ${ }^{\dagger}$ Department of Chemistry and Biochemistry, University of Arkansas, Fayetteville, AR 72701 \\ TCondensed Matter Physics and Materials Science Department, Brookhaven National \\ Laboratory, Upton, NY 11973
}

*Corresponding author: chenj@uark.edu (+1 479-575-6203) 
ABSTRACT: Controlling the deposition of exotic metals in the seeded growth of multi-metal nanostructures is challenging. This work describes a seeded growth method assisted by a mask for synthesis of segmented binary or ternary metal nanostructures. Silica is used as a mask to partially block the surface of a seed and a second metal is subsequently deposited on the exposed area, forming a bimetallic heterodimer. The initial demonstration was carried out on a Au seed, followed by deposition of Pd or Pt on the seed. It was found that Pd tends to spread out laterally on the seed while Pt inclines to grow vertically into branched topology on Au. Without removal of the $\mathrm{SiO}_{2}$ mask, $\mathrm{Pt}$ could be further deposited on the unblocked Pd of the Pd-Au dimer to form a Pt-Pd-Au trimer. The mask-assisted seeded growth provides a general strategy to construct segmented metallic nanoarchitectures.

KEYWORDS: Solid-State Chemistry, Nobel Metal Nanoparticles, Silica, Sol-Gel, Microemulsion. 


\section{INTRODUCTION}

Heterostructures of two or more metals with interfaces at the nanoscale is of particular significance because they exhibit unique properties and multi-functions distinctly different from the individual components. ${ }^{1-4}$ The diverse surface chemistry of these heteronanostructures enables new applications that are not possible with each component alone. For example, multisegmented metal nanorods have been demonstrated for applications in synergistic heterogeneous catalysis, ${ }^{5-6}$ self-electrophoretic nanomotors/ nanobatteries, ${ }^{7-8}$ multifunctional biomedicine, ${ }^{9-10}$ and multiplexed detections. ${ }^{11-12}$ To fabricate the heteronanostructures, sequential electrochemical deposition of metal ions into templates is the most common method since the 1990s. ${ }^{13-14}$ In this approach, commercially-available alumina or polycarbonate membranes with uniform pores are often used as templates, yielding segmented metal rods. ${ }^{15}$ This method could be further extended to selective growth of additional metals within the templates in solution after the initial electrochemical deposition of rod-shaped seeds. ${ }^{16-17}$ To generate heterostructures with other configurations, it is usually required to involve cost-intensive and time-consuming lithography techniques. ${ }^{18}$ In this work, a site-selective seeded growth method, termed mask-assisted seeded growth (MASG), is developed to expand the library of metal heterostructures with complex nanoarchitectures.

Seeded growth has emerged as a compelling method to create a wide variety of novel metal nanostructures. ${ }^{19-24}$ Conditions that yield heteronanostructures depend on a number of factors such as the structural characteristics of constituent components, the reduction kinetics of metal precursors, and the capping agents. For example, a high degree of lattice mismatch between the seed and the second metal prevents conformal growth of core@ shell structures and yields heteronanostructures of $\mathrm{Au}$ on $\mathrm{CoPt}_{3},{ }^{25} \mathrm{Au}$ rods on $\mathrm{Pt}$ cubes,${ }^{21}$ and $\mathrm{Cu}$ on $\mathrm{Au}^{24}$ Controlling the 
reduction kinetics can selectively direct the nucleation and subsequent growth of the second metal on the seed to form dimers of $\mathrm{Au}$ on $\mathrm{Pd}^{26}$ or other non-conformal structures such as $\mathrm{Ag} / \mathrm{Au}$ on Pd cubes ${ }^{27-28}$ and $\mathrm{Ag}$ on $\mathrm{Au}$ nanorods. ${ }^{29}$ On the other hand, blocking specific facets of the seeds by capping agents could lead to the growth of Rh on Pd heterostructures. ${ }^{30}$ Despite these advances, optimization of the growth conditions is largely material specific and it is beneficial to develop a general approach for the synthesis of multi-component heteronanostructures.

In this work, the MASG method uses $\mathrm{SiO}_{2}$ as a mask in seeded growth to partially block the surface of the seed and thus direct the deposition of exotic metals on the exposed surface of the seed, forming heteronanostructures. The asymmetric coating of $\mathrm{SiO}_{2}$ was demonstrated in the classical Stöber synthesis by using a polymeric ligand to partially block the diffusion of the solgel precursor to reach the surface of the nanoparticle. ${ }^{31}$ However, this method is only suitable for water-soluble nanoparticles. To overcome this limitation, the water-in-oil (W/O) microemulsion method is used to form the $\mathrm{SiO}_{2}$ mask. Unlike the classical Stöber synthesis of $\mathrm{SiO}_{2}$ coating, ${ }^{31-34}$ the W/O microemulsion confines the sol-gel condensation within the water droplets in bulk oil, particularly useful for the formation of $\mathrm{SiO}_{2}$ coating on nanoparticles suspended in hydrophobic solvents. ${ }^{35}$ By reducing the concentration of sol-gel precursor in the $\mathrm{W} / \mathrm{O}$ microemulsion, phase separation between the surface ligand and hydrolyzed precursor is created on the particle surface, leading to partial condensation of $\mathrm{SiO}_{2}$ on individual $\mathrm{Au}$ nanospheres to yield $\mathrm{Au}-\mathrm{SiO}_{2}$ dumbbell structures. Sequential reduction of Pd and Pt precursors on these dumbbell structures generates heterodimers of $\mathrm{Pd}-\mathrm{Au}$ and $\mathrm{Pt}-\mathrm{Au}$, and more complex $\mathrm{Pt}-\mathrm{Pd}-\mathrm{Au}$ heterotrimmers. The reaction mechanisms of the microemulsion for preparation of $\mathrm{Au}-\mathrm{SiO}_{2}$ dumbbells and their subsequent use for synthesis of heteronanostructures are elucidated. These heteronanostructures exhibit tunable optical properties in the visible markedly different from the individual components. 


\section{EXPERIMENTAL SECTION}

Chemicals. Hydrogen tetrachloroaurate trihydrate $\left(\mathrm{HAuCl}_{4} \cdot 3 \mathrm{H}_{2} \mathrm{O}, 99.99 \%\right)$, potassium tetrachloroplatinate $\left(\mathrm{K}_{2} \mathrm{PtCl}_{4}, 99.9 \%\right)$, potassium tetrachloropalladate $\left(\mathrm{K}_{2} \mathrm{PdCl}_{4}, 99.9 \%\right)$, and ammonium hydroxide $\left(28-30 \% \mathrm{NH}_{3} \cdot \mathrm{H}_{2} \mathrm{O}\right)$ were purchased from Alfa Aesar. Tetradecylamine (TDA, >95\%) was purchased from TCI. Tetraethoxysilane (TEOS, 98\%), poly(oxyethylene) nonylphenyl ether (Igepal CO-520), and polyvinylpyrrolidone (PVP, M.W. $=55,000)$ were purchased from Sigma-Aldrich. Cyclohexane (ACS grade) was purchased from EMD. All chemicals were used as received unless specified otherwise.

Synthesis of $\mathrm{Au}-\mathrm{SiO}_{2}$ Dumbbells. The $\mathrm{Au}-\mathrm{SiO}_{2}$ dumbbells were synthesized by controlling the hydrolysis and condensation of the $\mathrm{SiO}_{2}$ precursor during the coating process using $\mathrm{W} / \mathrm{O}$ microemulsions. Initially, $10-\mathrm{nm} \mathrm{Au}$ nanoparticles were prepared by reducing $\mathrm{HAuCl}_{4} \cdot 3 \mathrm{H}_{2} \mathrm{O}$ (19.7 mg, $0.05 \mathrm{mmol})$ in $5 \mathrm{~g}$ of TDA at $160{ }^{\circ} \mathrm{C}$ for $20 \mathrm{~min}$ under argon. After the reaction, the product was cooled to $100{ }^{\circ} \mathrm{C}$ and purified with toluene and ethanol to remove unreacted precursor and excess TDA. The Au nanoparticles were then dispersed in cyclohexane for silica coating in W/O microemulsions. In a typical procedure, $1 \mathrm{~mL}$ of $200 \mathrm{nM}$ Au nanoparticles was added to the mixture of $0.14 \mathrm{M}$ Igepal CO-520 in $40 \mathrm{~mL}$ cyclohexane in a round-bottom flask equipped with a magnetic stirring bar. Various amounts of TEOS $(20-200 \mu \mathrm{L})$ were added to the reaction mixture and allowed to mix for $30 \mathrm{~min}$ before the addition of $0.280 \mathrm{~mL}$ of $\mathrm{NH}_{3} \cdot \mathrm{H}_{2} \mathrm{O}$. The $\mathrm{Au}-\mathrm{SiO}_{2}$ dumbbell structures were formed when less than $50 \mu \mathrm{L}$ of TEOS was used. The reaction was allowed to proceed for $48 \mathrm{~h}$ and stopped by adding ethanol to interrupt the emulsion. The product was washed by ethanol and dispersed in $18 \mathrm{M} \Omega \mathrm{H}_{2} \mathrm{O}$ for future use.

Synthesis of Pd-Au Dimers. The Pd-Au dimers were synthesized by reducing the Pd precursor in the presence of $\mathrm{Au}-\mathrm{SiO}_{2}$ dumbbells using ascorbic acid. In a typical procedure, 8 
$\mathrm{mL}$ of $\mathrm{K}_{2} \mathrm{PdCl}_{4}$ was added, at a rate of $0.1 \mathrm{~mL} / \mathrm{min}$, to a $5 \mathrm{ml}$ aqueous solution containing Au$\mathrm{SiO}_{2}$ dumbbells $(\sim 15 \mathrm{pmol})$ and $\mathrm{PVP}(1 \mathrm{mg} / \mathrm{mL})$ at room temperature. Final concentrations of $\mathrm{K}_{2} \mathrm{PdCl}_{4}(0.06,0.2$, and $0.35 \mathrm{mM})$ in the reactions were used to yield $\mathrm{Pd}$ depositions with different thickness of $\sim 4, \sim 7$, and $\sim 10 \mathrm{~nm}$, respectively. The molar ratio of ascorbic acid to $\mathrm{K}_{2} \mathrm{PdCl}_{4}$ was kept at 10 to 1 for all three reactions. The product was washed by ethanol and dispersed in $18 \mathrm{M} \Omega \mathrm{H}_{2} \mathrm{O}$. The $\mathrm{SiO}_{2}$ component was then dissolved by mixing the $\mathrm{Pd}-\mathrm{Au}-\mathrm{SiO}_{2}$ particles overnight in an aqueous solution containing excess $\mathrm{NaOH}$ and PVP $(10 \mathrm{mg} / \mathrm{mL})$. The product was washed with water and dispersed in $18 \mathrm{M} \Omega \mathrm{H}_{2} \mathrm{O}$.

The thickness $\left(x_{P d}\right)$ of Pd deposition was estimated using a crude model which approximates the Pd layer is a semi-spherical shell interfacing with a spherical Au core. The radius of the semishell $\left(r_{P d}\right)$ can be derived from the average volume of $\mathrm{Pd}\left(\mathrm{nm}^{3}\right.$ per particle): $V_{P d}=\frac{1}{2}\left(\frac{4}{3} \pi r_{P d}^{3}-\frac{4}{3} \pi r_{A u}^{3}\right)$, where $r_{A u}$ is the radius of the Au core that can be measured from the transmission electron microscopy (TEM) image. The $V_{P d}$ was obtained from $\frac{V_{P d}}{V_{A u}}=\frac{C_{P d} a_{P d}^{3}}{C_{A u} a_{A u}^{3}}$, where $V_{A u}$ is the average volume of $\mathrm{Au}\left(\mathrm{nm}^{3}\right.$ per particle) assuming spherical shape $\left(V_{A u}=\frac{4}{3} \pi r_{A u}^{3}\right.$ ); $C_{P d}$ and $C_{A u}$ are the concentration of $\mathrm{Pd}$ and $\mathrm{Au}(\mathrm{mol} / \mathrm{L})$, respectively, which can be measured from the bulk sample; $a_{P d}$ and $a_{A u}$ are the lattice constant of Pd and Au (nm), respectively. The difference of the $r_{P d}$ and $r_{A u}$ gives the thickness of the Pd semi-shell: $x_{P d}=r_{P d}-r_{A u}$.

Synthesis of Pt-Au Dimers. The procedure for Pt-Au dimers was the same as that for Pd-Au dimers described above, with the exception that $\mathrm{K}_{2} \mathrm{PtCl}_{4}$ was used as the Pt precursor and final concentration of $\mathrm{Pt}$ in the reaction solution is $2.4 \mathrm{mM}$. 
Synthesis of Pt-Pd-Au Trimers. The Pt-Pd-Au trimmers were synthesized using a similar procedure as that for Pt-Au dimers except that the $\mathrm{Au}-\mathrm{SiO}_{2}$ was replaced by the pre-synthesized $\mathrm{Pd}-\mathrm{Au}-\mathrm{SiO}_{2}$ described above.

\section{RESULTS}

The MASG method has been demonstrated to synthesize bimetallic and trimetallic heteronanostructures. Figure 1A illustrates the general strategy of the MASG method using a Au seed and a second metal $\mathrm{Pd}$ as an example to create heterodimers. This method involves $\mathrm{SiO}_{2}$ to mask one side of a spherical nanoparticle and expose the other side for deposition of successive metals. After deposition, the $\mathrm{SiO}_{2}$ mask can be removed to yield heterodimers, trimers, or oligomers depending on the number of repeating depositions performed during the seeded growth. As an initial demonstration, Au and Pd were used as the seed and the second metal, respectively. The Au seeds prepared by reducing chloroauric acid in tetradecylamine were spherical nanoparticles with diameter of $9.7 \pm 1.3 \mathrm{~nm}$ (Fig. 1B). High resolution TEM (HRTEM) analysis shows that the Au seeds adopted decahedral geometry consisting of five tetrahedral subunits (Fig. S1). The subunits were bounded by $\{111\}$ crystal faces, arranging along a fivefold rotation axis along the [110] zone. ${ }^{36}$ Silica was subsequently condensed on these seeds using a modified W/O microemulsion method to form $\mathrm{Au}-\mathrm{SiO}_{2}$ dumbbells (Fig. 1C). The yield of dumbbells was close to $90 \%$ with half of the seed surface covered by $\mathrm{SiO}_{2}$. These $\mathrm{SiO}_{2}$ masked seeds were used for the deposition of Pd. Upon reducing Pd precursors with ascorbic acid, Pd was selectively deposited on the unblocked Au surface of these dumbbells to generate heterostructures of $\mathrm{Pd}-\mathrm{Au}-\mathrm{SiO}_{2}$ (Fig. 1D). Removal of the $\mathrm{SiO}_{2}$ mask yielded $\mathrm{Pd}-\mathrm{Au}$ heterodimers (Fig. 1E). 


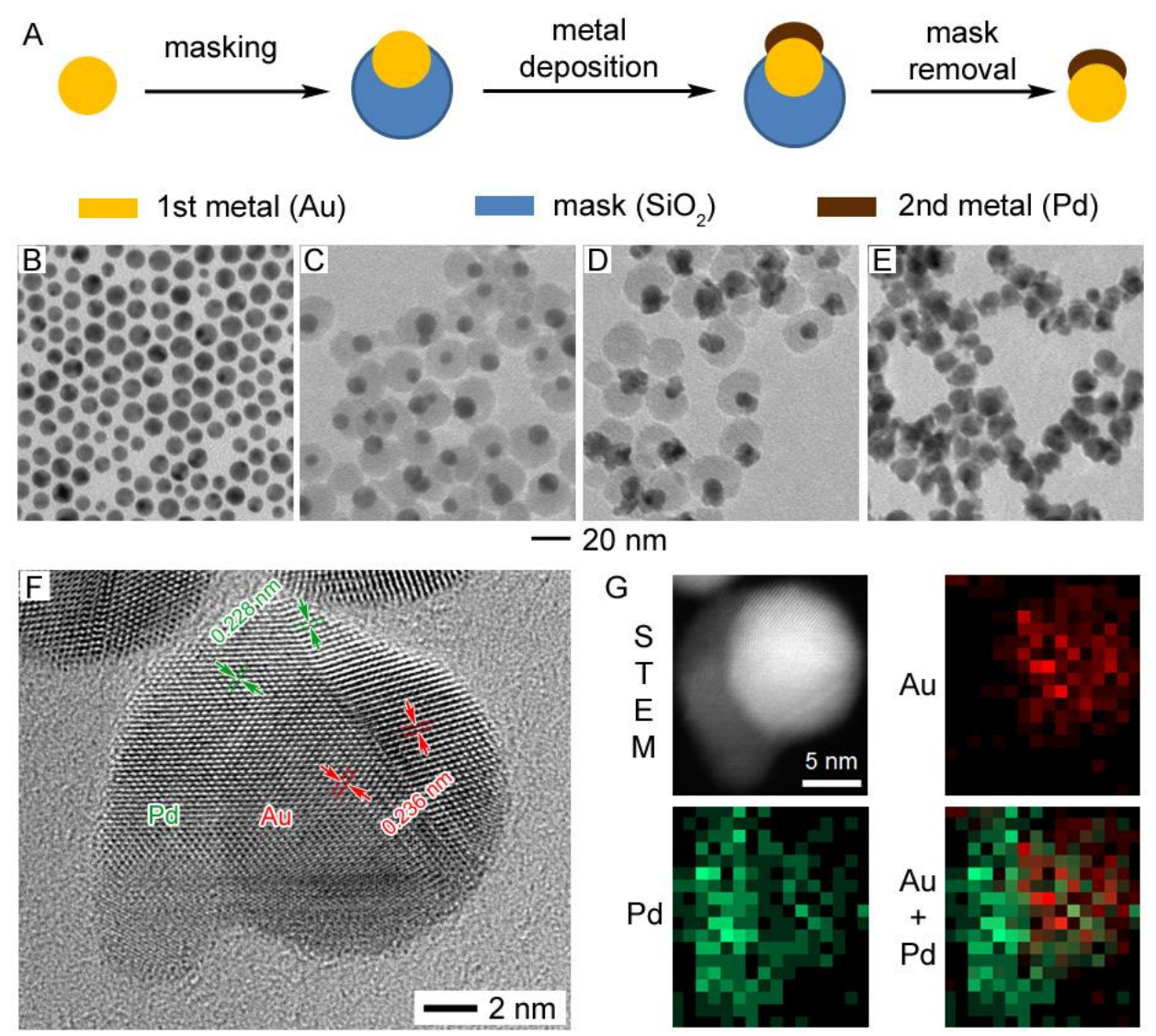

Figure 1. (A) Schematic illustration of the MASG method for synthesis of metallic heteronanostructures. TEM characterization of products from each step in the process using $P d$ $A u$ as a demonstration: (B) Au spherical nanoparticles with a mean diameter of $9.7 \pm 1.3 \mathrm{~nm}$; $(C)$ $\mathrm{Au}$-SiO $\mathrm{O}_{2}$ dumbbell nanoparticles; (D) $\mathrm{Pd}$-Au-SiO ${ }_{2}$ nanoparticles; and (E) Pd-Au heterodimers. TEM characterization of an individual Pd-Au heterodimer: $(F)$ HRTEM image and $(G) H A A D F$ STEM image and EDX elemental mapping.

The Pd-Au dimers were further characterized by HRTEM, high angle annular dark field scanning transmission electron microscopy (HAADF-STEM), and energy-dispersive X-ray spectroscopy (EDX). The HRTEM image of individual Pd-Au dimers clearly shows that the two components were assembled into heterostructures (Fig. 1F). The Z-contrast HAADF-STEM image and EDX mapping indicate that the dimers adopted a mushroom configuration with a $\mathrm{Au}$ spherical stem and a Pd cap (Fig. 1G). Further analysis of the HRTEM image shows the continuation of lattice fringes from $\mathrm{Au}$ to $\mathrm{Pd}$. The lattice spacing was measured to be 0.236 and $0.228 \mathrm{~nm}$ corresponding to the spacing of $\{111\}$ planes of $\mathrm{Au}$ and $\mathrm{Pd}$, respectively. This result suggests an 
epitaxial growth of Pd on the $\{111\}$ facets of decahedral five-fold twinned Au nanoparticles, in agreed with the previous in situ TEM observations. ${ }^{37}$ Without the $\mathrm{SiO}_{2}$ mask, no $\mathrm{Au}-\mathrm{Pd}$ dumbbells were found in the product under identical conditions (Fig. S2).

The growth of $\mathrm{Pd}$ on $\mathrm{Au}-\mathrm{SiO}_{2}$ dumbbells could be manipulated by varying the amount of $\mathrm{Pd}$ precursor introduced during the deposition onto the $\mathrm{Au}-\mathrm{SiO}_{2}$ seeds. The amount of $\mathrm{Au}-\mathrm{SiO}_{2}$ seeds was kept constant and the concentration of $\mathrm{Au}-\mathrm{SiO}_{2}$ was estimated using the extinction coefficient at $520 \mathrm{~nm}\left(\varepsilon_{A u}=(1.55 \pm 0.01) \times 10^{8} \mathrm{M}^{-1} \cdot \mathrm{cm}^{-1}\right.$, Fig. S3 $)$. To maintain the same reaction volume, different concentrations of $\mathrm{K}_{2} \mathrm{PdCl}_{4}$ were added to the reaction containing 15 $p$ mol of $\mathrm{Au}_{-} \mathrm{SiO}_{2}$ at a rate of $0.1 \mathrm{~mL} / \mathrm{min}$. Because the underpotential deposition of $\mathrm{Pd}$ on $\mathrm{Au}$ favors heterogeneous nucleation of $\mathrm{Pd}$ on $\mathrm{Au}$ over $\mathrm{Pd}$ on $\mathrm{Pd}{ }^{38-39} \mathrm{Pd}$ preferentially nucleates laterally on the Au surface to cover the entire area of the exposed Au prior to the growth on newly-deposited $\mathrm{Pd}$. Increased concentration of $\mathrm{K}_{2} \mathrm{PdCl}_{4}$ from 0.06 , to 0.20 and $0.35 \mathrm{mM}$ resulted in thicker deposition of $\mathrm{Pd}$, as shown in Figure 2A, 1D, and 2B, respectively. A crude model was established to calculate the deposition thickness of Pd by approximating the Pd layer as a semi-spherical shell interfacing with a spherical core of $\mathrm{Au}$ as described in the experimental section. The deposition thickness of Pd was estimated to be 4, 7, $10 \mathrm{~nm}$ for the three samples, denoted as $s \mathrm{Pd}-\mathrm{Au}-\mathrm{SiO}_{2}, m \mathrm{Pd}-\mathrm{Au}-\mathrm{SiO}_{2}, l \mathrm{Pd}-\mathrm{Au}-\mathrm{SiO}_{2}$, respectively.

Careful control of the reduction rate of the second metal is important to ensure heterogeneous nucleation and subsequent growth. A mild reduction of Pd was maintained for the deposition of Pd on Au using ascorbic acid as a reducing agent. The correlation between the amount of Pd formed and the amount of Pd precursor added was plotted in Figure 2C. In this study, the amount of ascorbic acid was in excess, 10:1 equivalent to the Pd precursor. The amount of deposited Pd on Au was linearly proportional to the amount of Pd precursor added to the reaction 
in the range of molar ratios of $\mathrm{Pd}$ to $\mathrm{Au}$ less than 10. The slope of the linear fitting curve was $0.90 \pm 0.04$, suggesting that nearly all the $\mathrm{Pd}$ precursor was reduced under these reaction conditions. The XRD results also show an increase of the $\mathrm{Pd}$-to-Au ratio from $s \mathrm{Pd}-\mathrm{Au}-\mathrm{SiO}{ }_{2}$, to $m \mathrm{Pd}-\mathrm{Au}-\mathrm{SiO}_{2}$ and $l \mathrm{Pd}-\mathrm{Au}-\mathrm{SiO}_{2}$ by comparing the ratio of (111) peaks of $\mathrm{Pd}$ to $\mathrm{Au}$ in Figure 2D. Diffusion between the two metals at the interface may occur, ${ }^{40}$ however, the rate could be rather slow at room temperature. ${ }^{41-42}$
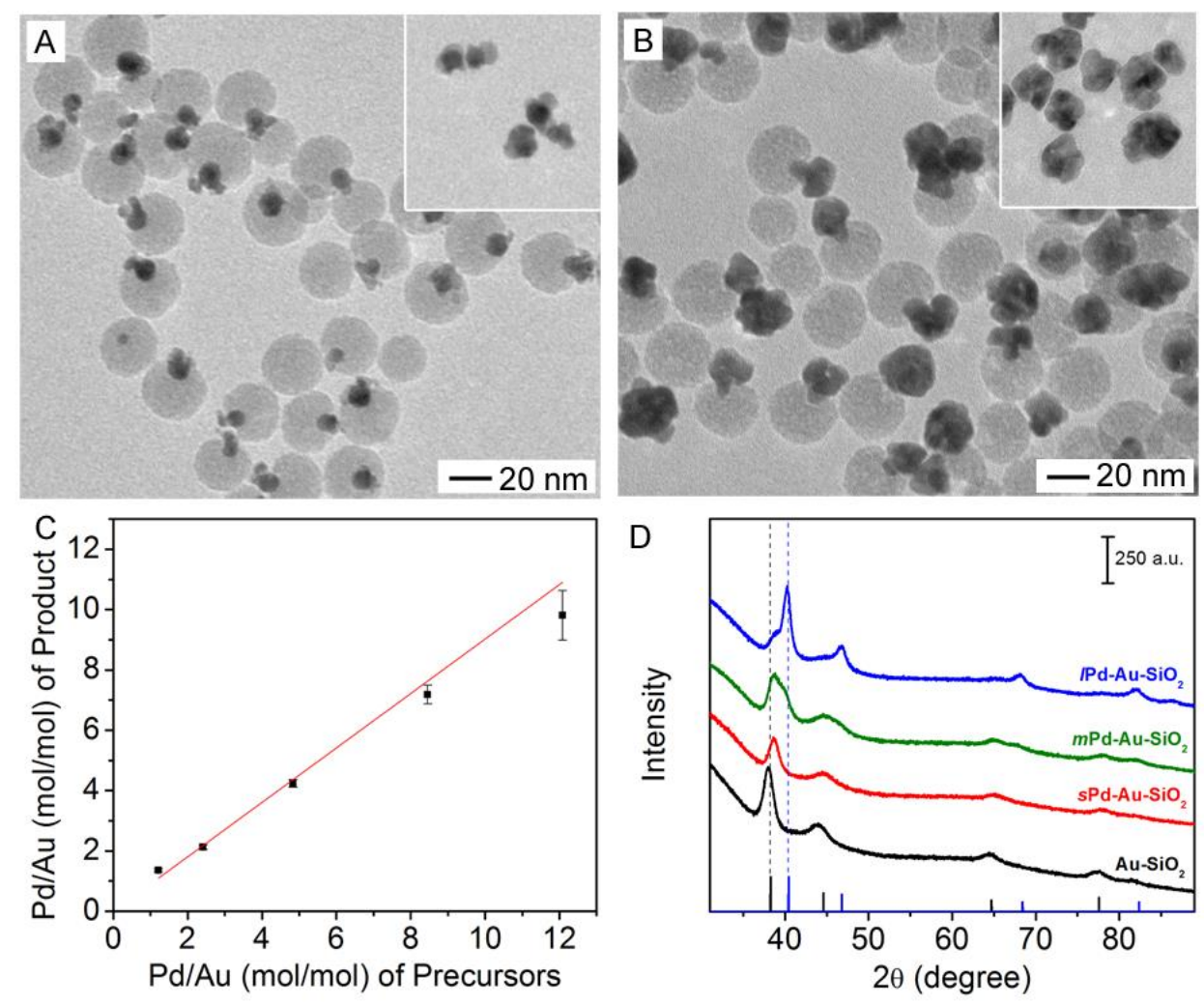

Figure 2. TEM characterization of $\mathrm{Pd}-\mathrm{Au}$-SiO $\mathrm{O}_{2}$ heteronanostructures with different thickness of Pd deposition: (A) 4; and (B) $10 \mathrm{~nm}$. The insets show the corresponding TEM images of Pd-Au heterodimers after the removal of silica. $(C)$ Plot of the relative quantities of $P d$ in the dimers versus that in the precursor. (D) XRD patterns of the Au-SiO 2 dumbbell nanoparticles $\left(\mathrm{Au}-\mathrm{SiO} \mathrm{O}_{2}\right)$ and $\mathrm{Pd}-\mathrm{Au}-\mathrm{SiO}_{2}$ heteronanostructures with Pd deposition thickness of 4, 7, and $10 \mathrm{~nm}$, denoted as $\mathrm{sPd}-\mathrm{Au}-\mathrm{SiO}_{2}, \mathrm{mPd}-\mathrm{Au}-\mathrm{SiO}_{2}, \mathrm{PPd}-\mathrm{Au}-\mathrm{SiO}_{2}$, respectively.

The deposition of Pd on Au could be monitored by the change of the optical properties of the resultant heteronanostructures. Figure 3A shows the UV-Vis spectra of $s \mathrm{Pd}-\mathrm{Au}-\mathrm{SiO}_{2}, m \mathrm{Pd}-\mathrm{Au}-$ $\mathrm{SiO}_{2}$ and $l \mathrm{Pd}-\mathrm{Au}-\mathrm{SiO}_{2}$. Due to the damping effects of $\mathrm{Pd}$ on the surface plasmon resonance of 
$\mathrm{Au},{ }^{43}$ the extinction peak of Au nanoparticles at $520 \mathrm{~nm}$ was gradually flattened out as the deposition volume of Pd increased The optical spectra were further simulated using the discrete dipole approximation (DDA) method, ${ }^{44}$ based on our crudely modelled geometry shown in the insets of Figure 3, B-D. Due to small size of the particles, light absorption dominated over scattering for their surface plasmon resonances. The simulated spectra matched well with the empirical extinction spectra, showing a decrease in extinction efficiency of Au at $520 \mathrm{~nm}$ after deposition of Pd, from 0.652 (Fig. S4) to 0.420 (Fig. 3B), by about one third. As the size of Pd increases from a $x_{P d}$ of $4 \mathrm{~nm}$ to $7 \mathrm{~nm}$ and $10 \mathrm{~nm}$, the extinction efficiency at $520 \mathrm{~nm}$ slightly increases from 0.420 , to 0.470 and 0.550 , respectively. The corresponding extinction cross sections of individual dimers increase from $0.75 \times 10^{-16}$, to $1.40 \times 10^{-16}$ and $2.50 \times 10^{-16} \mathrm{~m}^{2}$. The optical spectra of another possible model of the Au-Pd dimer containing the same amount of $\mathrm{Au}$ and Pd by volume were also calculated. In this second model, the Au sphere is covered by a partial ellipsoid, as shown in Figure S5. The calculated spectra of the second model are very similar to the semi-spherical Pd shell model, suggesting that the optical spectra of the Au-Pd dimer are insensitive to certain details of the configuration such as the flat cutoff of a half-shell versus the curvature of an ellipsoid. By observing the change in the spectral shapes and intensity at $520 \mathrm{~nm}$, the colorimetric method provides a simple and convenient means to track the deposition thickness of Pd in situ. 

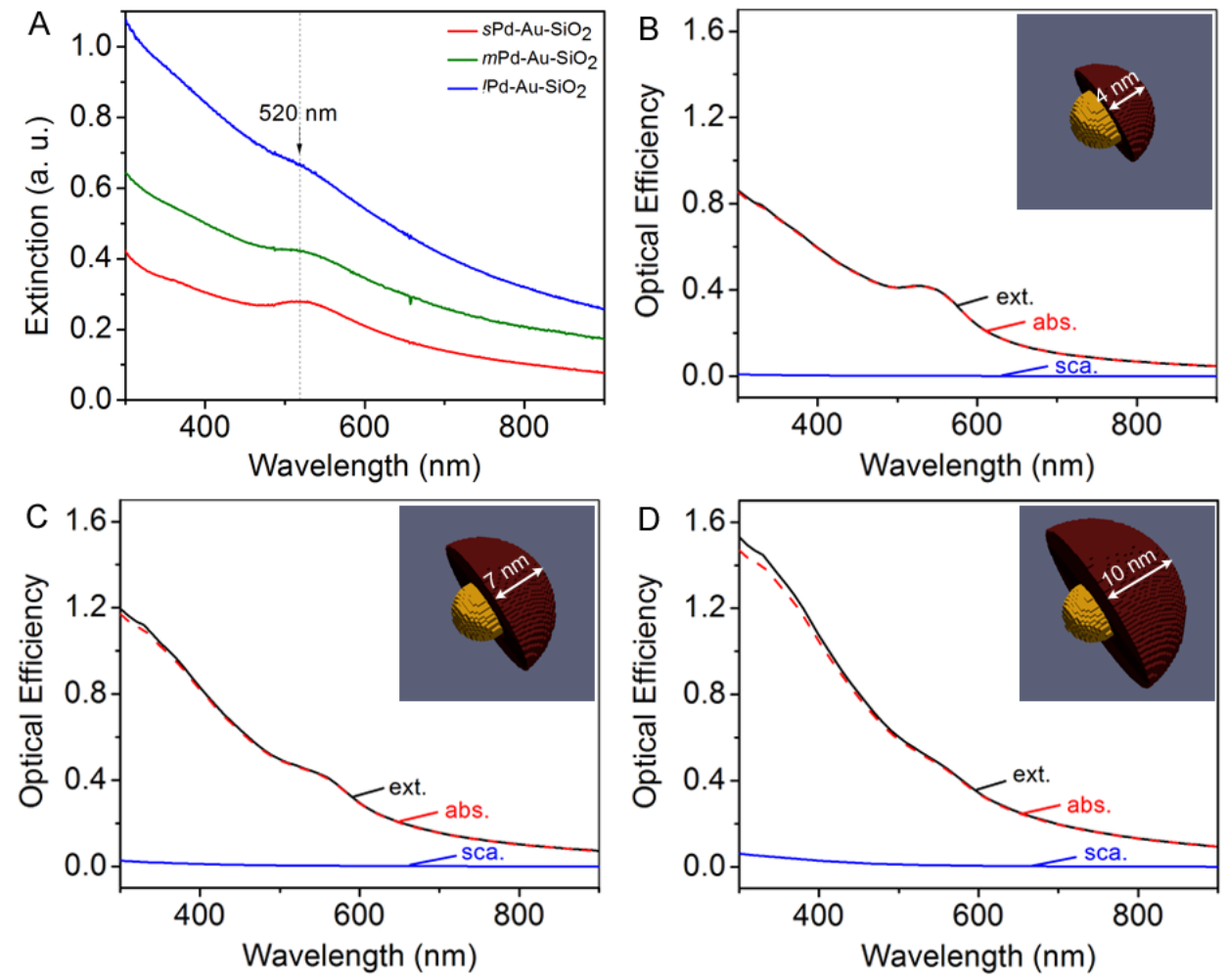

Figure 3. $\mathrm{UV}$-Vis spectra of the $\mathrm{Pd}$-Au-SiO $\mathrm{S}_{2}$ heteronanostructures with a Pd deposition thickness of 4, 7, and $10 \mathrm{~nm}$ on Au nanoparticles with a diameter of 9.7 $\pm 1.3 \mathrm{~nm}$, denoted as $\mathrm{sPd}-\mathrm{Au}-\mathrm{SiO}$, $\mathrm{mPd}-\mathrm{Au}-\mathrm{SiO}_{2}$, and $\mathrm{lPd}-\mathrm{Au}-\mathrm{SiO}_{2}$, respectively. DDA simulation of the optical spectra of Pd-Au heterodimers with a Pd thick deposition of 4, 7, and $10 \mathrm{~nm}$ on a Au nanosphere with a diameter of $10 \mathrm{~nm}$, denoted as $s P d-A u(B), m P d-A u(C), l P d-A u(D)$, respectively. The insets show the geometries of the Pd-Au heterodimers used for the simulation.

To further evaluate the versatility of the MASG method, Pt was examined as a second metal for deposition on the $\mathrm{Au}-\mathrm{SiO}_{2}$ dumbbells. Interestingly, $\mathrm{Pt}$ was deposited on $\mathrm{Au}$ as a dendritic configuration branching out vertically as opposed to the lateral growth of $\mathrm{Pd}$ on $\mathrm{Au}$ (Fig. 4). Further analysis of HRTEM images shows the lattice spacing was measured to be 0.236 and $0.228 \mathrm{~nm}$ corresponding to the spacing of $\{111\}$ planes of $\mathrm{Au}$ and $\mathrm{Pt}$, respectively. It is suggested that Pt clusters could be deposited on $\mathrm{Au}$ seeds through either direct reduction or oriented attachment on the $\{111\}$ facets of decahedral five-fold twinned Au nanoparticles. ${ }^{45-46}$ After the initial epitaxial growth of Pt on $\mathrm{Au}$, growth of Pt on Pt is more favorable than that on $\mathrm{Au}$ possibly due to the overpotential of $\mathrm{Pt}$ deposition on the $\mathrm{Au}$ surface. The slow reduction 
kinetics promotes the growth of Pt along the $\langle 111\rangle$ direction into branched structures. ${ }^{47}$ The HAADF-STEM image and EDX elemental mapping verified the composition of $\mathrm{Au}$ and $\mathrm{Pt}$ heterodimer structures.
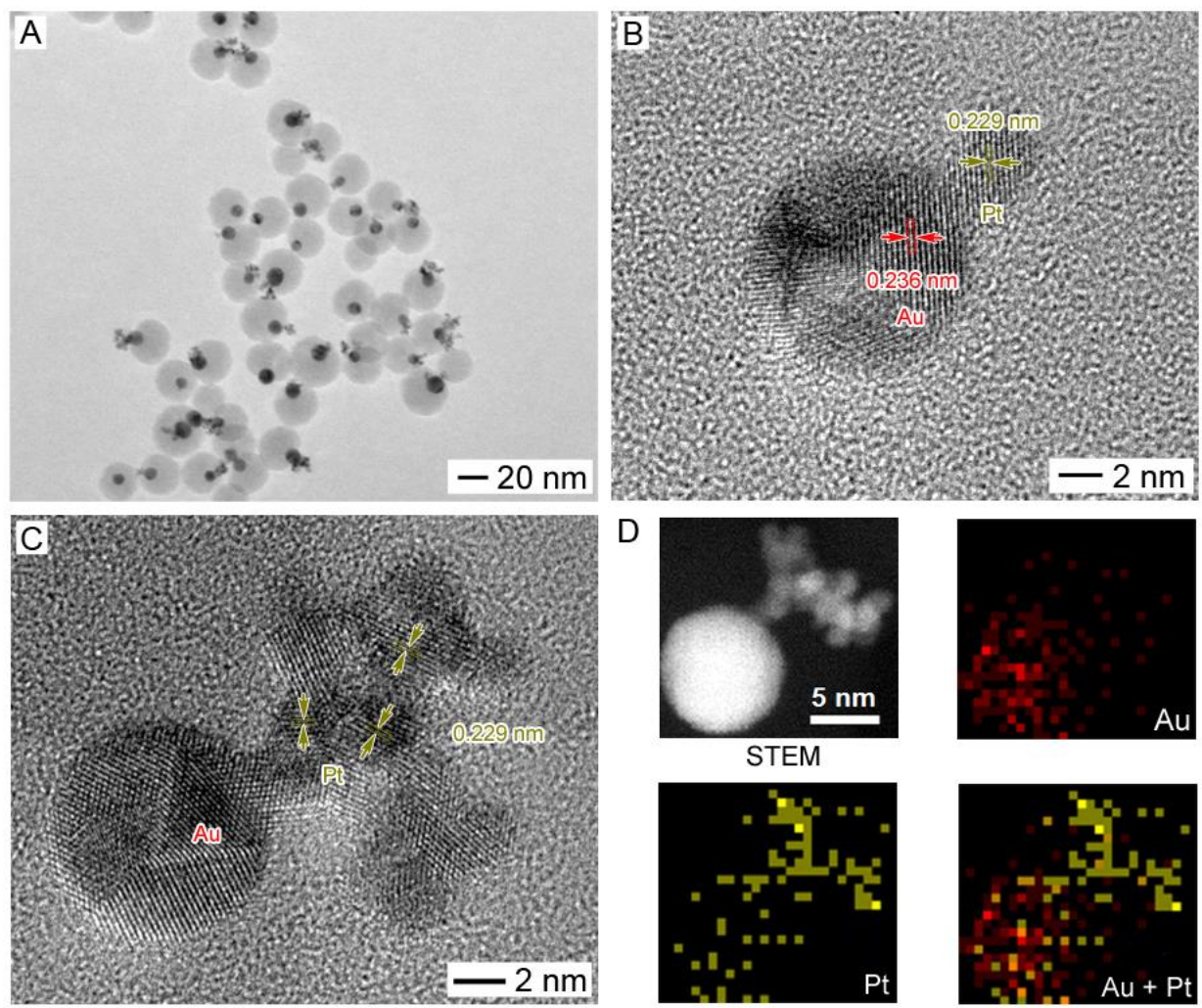

Figure 4. TEM characterization of Pt-Au-SiO ${ }_{2}$ heterostructures: (A) overview image; (B, C) HRTEM of individual particles; and (D) HAADF-STEM image and EDX elemental mapping.

The MASG method can be further extended to grow an additional component at a designated site of the heterodimers using the $\mathrm{SiO}_{2}$ masking strategy. As a demonstration, $\mathrm{Pt}$ was added with ascorbic acid to the suspension of $\mathrm{Pd}-\mathrm{Au}-\mathrm{SiO}_{2}$ heterostructures with $\mathrm{Au}$ protected by the $\mathrm{SiO}_{2}$ coating. Dendritic Pt caps were formed on the Pd side of the Pd-Au dimers as shown in Figure 5, in contrast to equal deposition on both $\mathrm{Pd}$ and $\mathrm{Au}$, which was observed on the unprotected dimers (Fig. S6). It is hard to differentiate Pd and Pt by comparing the lattice spacing of $\{111\}$ facets because their lattice constant are almost identical $\left(0.389 \mathrm{~nm}\right.$ for Pd versus 0.392 for Pt). ${ }^{48}$ However, the vertical growth pattern of Pt is distinctly different from the lateral one of Pd. From 
the HRTEM analysis, it was found that epitaxial growth was extended from $\mathrm{Pd}$ on $\mathrm{Au}\{111\}$ faces to $\mathrm{Pt}$ on $\mathrm{Pd}\{111\}$ faces of $\mathrm{Pd}-\mathrm{Au}$. The composition of $\mathrm{Au}, \mathrm{Pd}$, and $\mathrm{Pt}$ of individual heterotrimers was further confirmed by the HAADF-STEM image and EDX elemental mapping.
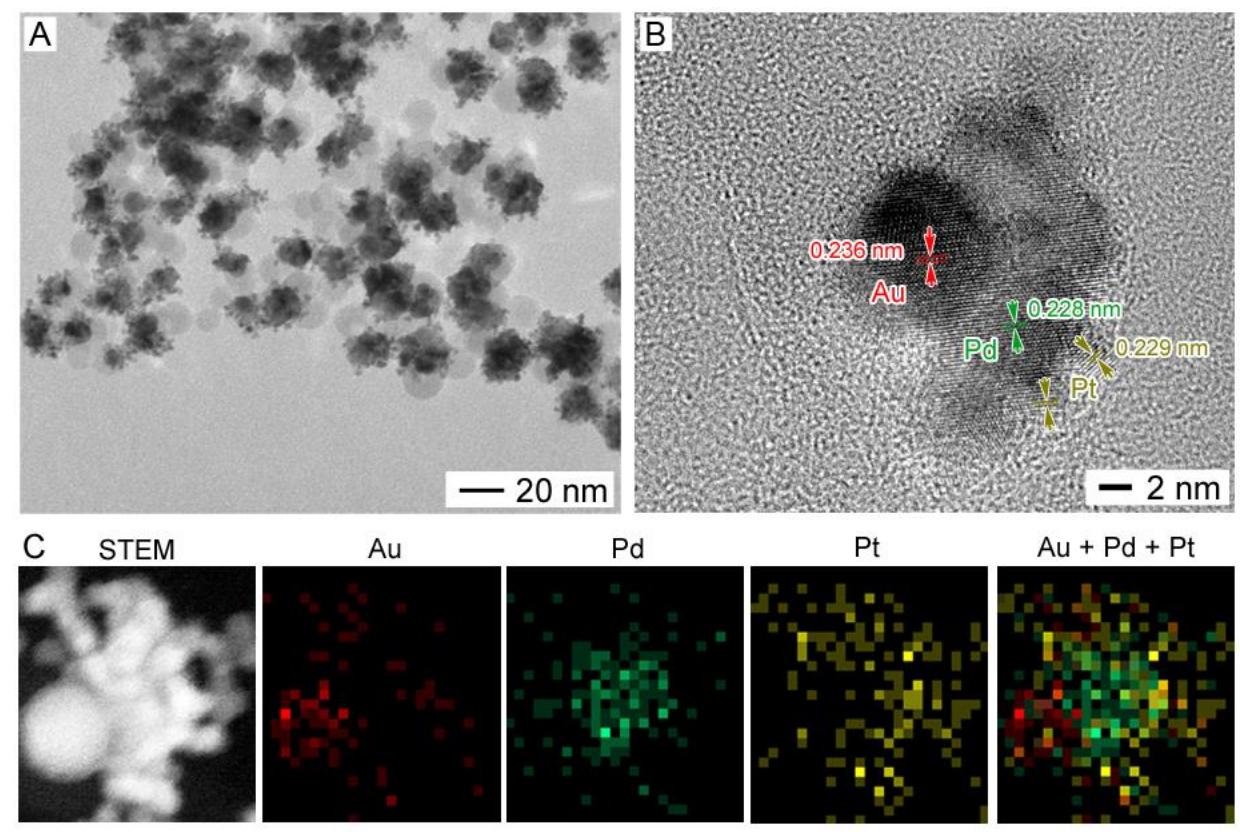

Figure 5. TEM characterization of Pt-Pd-Au-SiO ${ }_{2}$ heteronanostructures: (A) overview image; (B) HRTEM of an individual particle; (C) HAADF-STEM image and EDX elemental mapping.

\section{DISCUSSION}

The formation of metal- $\mathrm{SiO}_{2}$ dumbbell nanoparticles is the key to the success of the MASG method. A high yield of $\mathrm{Au}-\mathrm{SiO}_{2}$ dumbbells was accomplished by a modified W/O microemulsion method. In a typical W/O microemulsion, the silica coating on a nanoparticle is formed by hydrolysis and condensation of TEOS within a micrometer- or nanometer-sized droplet that is generated in a homogeneous mixture of water, organic solvent (oil), and surfactant. The conventional method often results in core@shell structures with minimal inhomogeneity in the coating. ${ }^{35}$ The modified W/O microemulsion was carried out by hydrolysis and condensation of TEOS on TDA-coated Au nanoparticles using ammonia in the presence of surfactant (Igepal CO-520) and organic solvent (cyclohexane). To increase the yield of $\mathrm{Au}-\mathrm{SiO}_{2}$ 
dumbbells, the concentration of TEOS was reduced to create phase separation between TDA and hydrolyzed TEOS on the surface of Au nanoparticles.
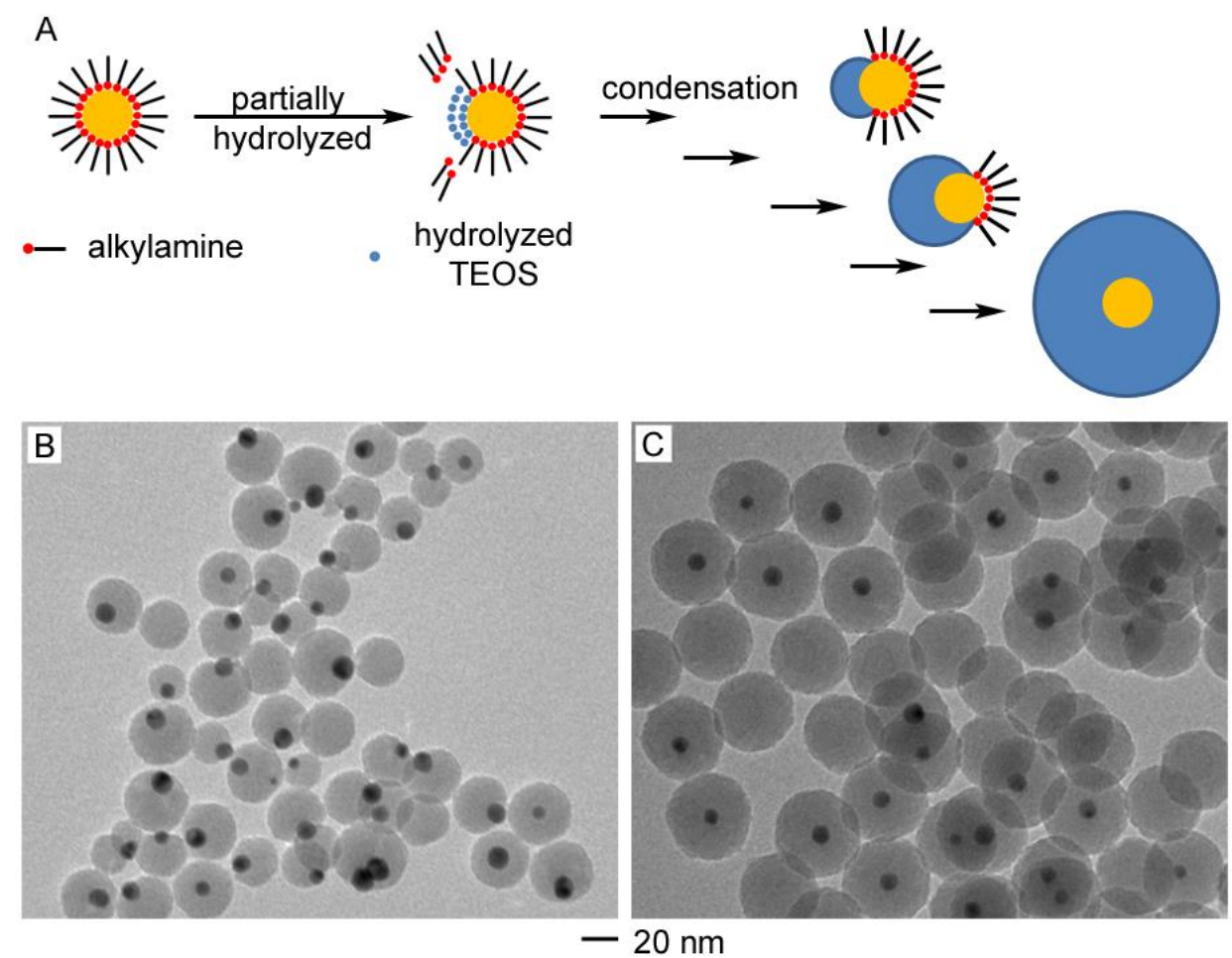

Figure 6. (A) Schematic illustration of the mechanism of silica coating via a micro-emulsion process. TEM characterization of the corresponding samples in the proposed mechanism using two different concentrations of TEOS under identical reaction conditions: (B) at low concentration (3.4 $\mathrm{mM})$; and $(\mathrm{C})$ at high concentration $(22.4 \mathrm{mM})$.

The mechanism based on phase separation in the W/O microemulsion was illustrated in Figure 6A. Initially, TDA ligands on the nanoparticles were partially replaced by the hydrolyzed TEOS, resulting in phase segregation of hydrophobic oleylamine and hydrophilic TEOS. ${ }^{49}$ Further condensation of TEOS was limited to the hydrophilic portion of the particle surface, yielding dumbbell structures of $\mathrm{Au}-\mathrm{SiO}_{2}$ (Fig. 6B). As the concentration of TEOS increases, more TDA ligands are substituted by the hydrolyzed TEOS, leading to a gradual expansion of TEOS to encompass the entire surface of the Au nanoparticles and thus further condensation to form complete $\mathrm{SiO}_{2}$ shell (Fig. 6C). The resultant $\mathrm{SiO}_{2}$ is porous, allowing small molecules to 
diffuse in and out more easily as compared to crystalline materials. For example, water can diffuse through the pores of $\mathrm{SiO}_{2}$ to reach the surface of $\mathrm{Au}$ nanoparticles, resulting in a $\mathrm{SiO}_{2}$ shell filled with water. Assuming that a simple mathematic average can be used to estimate the dielectric constant of a two-component mixture, the refractive index of porous $\mathrm{SiO}_{2}$ surrounding $\mathrm{Au}$ nanoparticles can be shown to be very similar to that of toluene. This was confirmed by measuring the extinction spectra of $\mathrm{Au}$ nanoparticles in toluene and $\mathrm{Au}-\mathrm{SiO}_{2}$ in water. The spectra are very similar and consistent with our approximation (Fig. S7). Despite of its porous nature, the $\mathrm{SiO}_{2}$ mask can act as a protecting group and effectively reduce the surface accessibility of reactant molecules in a chemical reaction. To verify the protecting function of $\mathrm{SiO}_{2}$, the reactivity of $\mathrm{Au}$ in the dumbbells and the core@shell structures was compared using two model reactions: an etching experiment using excess $\mathrm{KCN}$ and the reduction of $p$ nitrophenol by excess $\mathrm{NaBH}_{4}$.

The reactivity of the Au cores in the dumbbells is markedly different from that of core-shell structures because $\mathrm{SiO}_{2}$ coating largely blocks the diffusion of reactants. The etching of $\mathrm{Au}$ involves the use of $\mathrm{KCN}$ in aqueous solution as follows: $4 \mathrm{Au}+8 \mathrm{CN}^{-}+\mathrm{O}_{2}+2 \mathrm{H}_{2} \mathrm{O} \rightarrow$ $4\left[\mathrm{Au}(\mathrm{CN})_{2}\right]^{-}+4 \mathrm{OH}^{-}$. The rate of the reaction is mainly limited by the diffusion of $\mathrm{CN}^{-}$through the pores of $\mathrm{SiO}_{2}$ because the dissolved $\mathrm{O}_{2}$ and water molecules have covered the surface of $\mathrm{Au}$. As the size of $\mathrm{Au}$ nanoparticles decreases during the etching process, the extinction efficiency reduces linearly while cross-section decreases exponentially (Fig. S6). The dissolution of Au could then be monitored by the diminished rate of extinction at $520 \mathrm{~nm}$ (Fig. 7A). It was found that the etching rate of $\mathrm{Au}$ in the dumbbell particles was faster than that in the core@shell structures. In the case of core@ shell structures, the etching rate was further slowed down after 10 min, possibly because the slower diffusion rate of the larger $\left[\mathrm{Au}(\mathrm{CN})_{2}\right]^{-}$anions further slows 
down the etching rate of $\mathrm{Au}$. After dissolution of $\mathrm{Au}$, the dumbbell particles yielded half shells of silica while complete silica shells were found as the product for core@ shell particles (Fig. 7, B and $\mathbf{C}$ ). The slight change of $\mathrm{SiO}_{2}$ morphology after etching could be possibly due to the structure reconstruction in the basic solution during etching process. ${ }^{50}$
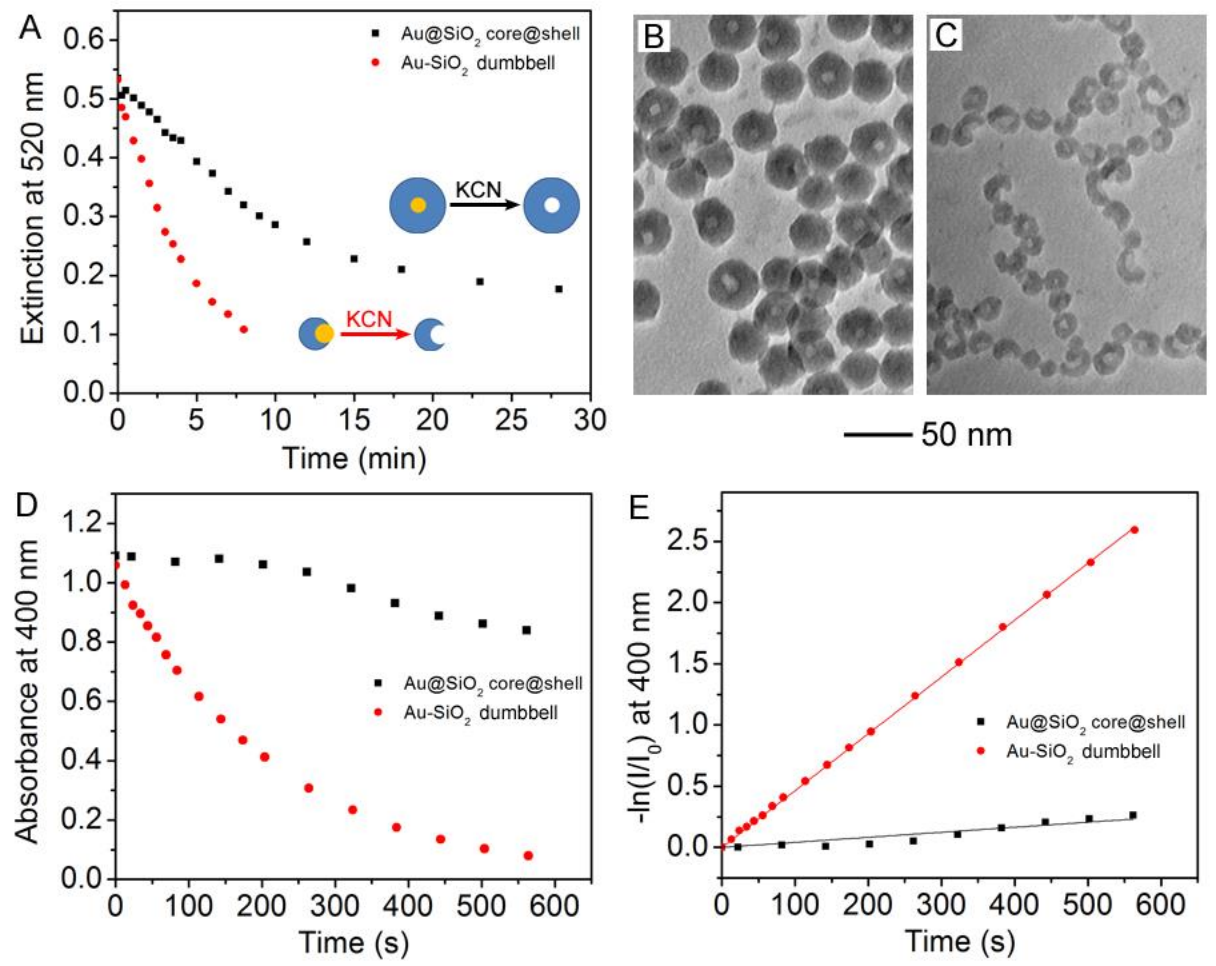

Figure 7. Etching process of Au nanoparticles: (A) Plot of extinction at $520 \mathrm{~nm}$ as a function of etching time; TEM images of Au@SiO $\mathrm{S}_{2}$ core@shell structures $(B)$ and Au-SiO $\mathrm{O}_{2}$ dimers $(C)$ after etching. Kinetic study of 4-nitrophenol reduction on Au-SiO ${ }_{2}$ nanostructures: (D) plot of normalized absorbance $\left(I / I_{0}\right)$ of p-nitrophenolate ion at $400 \mathrm{~nm}$ as a function of time for different catalysts; and $(E)$ plot of $-\ln \left(I / I_{0}\right)$ as a function of time for different catalysts. The solid lines are the linear fits of the data and the slope gives $k_{\text {app }}$ of the reaction.

The accessibility of $\mathrm{SiO}_{2}$-masked surface is much more difficult for the deposition of additional metals because the reduction typically involves two reactants: a relatively-large anion of transition metal complex and ascorbic acid. The assumption was examined by a model reaction involving two reactants, the reduction of $p$-nitrophenol by excess $\mathrm{NaBH}_{4}$. The same amounts of dumbbell particles and core@shell particles were used to catalyze the $p$-nitrophenol 
reduction. The reaction was monitored by decreased intensity of absorbance at $400 \mathrm{~nm}$ corresponding to the disappearance of $p$-nitrophenol (Fig. 7D). The reduction could be treated as a pseudo first order reaction when $\mathrm{NaBH}_{4}$ was in large excess. ${ }^{51}$ The data were then plotted as first order with respect to $p$-nitrophenol (Fig. 7E). The rate constant of $p$-nitrophenol reduction on the dumbbell particles was found to be $(4.64 \pm 0.01) \times 10^{-3} \mathrm{~s}^{-1}$, more than 10 fold faster compare to the core@shell structures $\left.(4.11 \pm 0.32) \times 10^{-4} \mathrm{~s}^{-1}\right)$. In fact, the $p$-nitrophenol reduction on the core@shell particles deviates from the first order kinetics, suggesting that the concentration of $\mathrm{NaBH}_{4}$ is insufficient to assume pseudo first order reaction. This result, in turn, implies that the diffusion rates of reactants are significantly reduced. Both the etching reaction of $\mathrm{Au}$ and the $p$ nitrophenol reduction have demonstrated that the $\mathrm{SiO}_{2}$ coating indeed serve as a mask to block chemical reactions.

\section{CONCLUSION}

The MASG method has been developed using $\mathrm{SiO}_{2}$ as a mask to partially protect the surface of seeds from further reaction and subsequently applied to synthesize $\mathrm{Pd}-\mathrm{Au}$ and $\mathrm{Pt}-\mathrm{Au}$ heterodimers and Pt-Pd-Au heterotrimers. The initial $\mathrm{Au}-\mathrm{SiO}_{2}$ dumbbell structures could be readily synthesized by a modified $\mathrm{W} / \mathrm{O}$ microemulsion due to the phase separation of hydrophobic surface ligands and hydrophilic hydrolyzed TEOS. Further epitaxial growth of Pd and $\mathrm{Pt}$ on $\mathrm{Au}$ was observed. Interestingly, $\mathrm{Pd}$ is apt to laterally grow on $\mathrm{Au}$ by capping the unblocked Au surface while Pt prefers to grow vertically into branched structures after initial deposition on Au. Replacing the noble metal with a $3 d$ transition metal as the second metal, the

active metal could subsequently be oxidized or sulfurized to oxide or sulfide. ${ }^{52-53}$ Therefore, the MASG method is potentially a versatile strategy to generate hybrid heteronanostructures with 
multi-components for improving the existing applications and finding new uses in many areas related to energy conversion and human health.

\section{Supporting Information}

Experimental details, TEM characterization of $\mathrm{Au}$ nanoparticles and Pt-Pd-Au heteronanostructures synthesized without protection of $\mathrm{SiO}_{2}, \mathrm{UV}-\mathrm{Vis}$ spectra of $\mathrm{Au}$ nanoparticles and $\mathrm{Au}-\mathrm{SiO}_{2}$ dumbbells, as well as DDA simulation of optical spectra of individual $\mathrm{Au}$ nanospheres with different diameters. This material is available free of charge via the Internet at http://pubs.acs.org.

\section{Acknowledgement}

This work was supported in part by the Ralph E. Powe Jr. Faculty Enhancement Award, funds from Arkansas Bioscience Institute, and startup funds from the University of Arkansas to J.C. The work done at Brookhaven National Laboratory was supported by the US Department of Energy, Basic Energy Sciences, by the Materials Sciences and Engineering Division under Contract No. DE-AC02-98CH10886. 


\section{References:}

(1) Ferrando, R.; Jellinek, J.; Johnston, R. L. Nanoalloys: From Theory to Applications of Alloy Clusters and Nanoparticles. Chem. Rev. 2008, 108, 845-910.

(2) Zheng, H.; Li, Y.; Liu, H.; Yin, X.; Li, Y. Construction of Heterostructure Materials toward Functionality. Chem. Soc. Rev. 2011, 40, 4506-4524.

(3) Walther, A.; Müller, A. H. E. Janus Particles: Synthesis, Self-Assembly, Physical Properties, and Applications. Chem. Rev. 2013, 113, 5194-5261.

(4) Buck, M. R.; Schaak, R. E. Emerging Strategies for the Total Synthesis of Inorganic Nanostructures. Angew. Chem. Int. Ed. 2013, 52, 6154-6178.

(5) Liu, F.; Lee, J. Y.; Zhou, W. Template Preparation of Multisegment PtNi Nanorods as Methanol Electro-Oxidation Catalysts with Adjustable Bimetallic Pair Sites. J. Phys. Chem. B 2004, 108, 17959-17963.

(6) Liu, F.; Lee, J. Y.; Zhou, W. J. Multisegment PtRu Nanorods: Electrocatalysts with Adjustable Bimetallic Pair Sites. Adv. Funct. Mater. 2005, 15, 1459-1464.

(7) Paxton, W. F.; Kistler, K. C.; Olmeda, C. C.; Sen, A.; St. Angelo, S. K.; Cao, Y.; Mallouk, T. E.; Lammert, P. E.; Crespi, V. H. Catalytic Nanomotors: Autonomous Movement of Striped Nanorods. J. Am. Chem. Soc. 2004, 126, 13424-13431.

(8) Liu, R.; Sen, A. Autonomous Nanomotor Based on Copper-Platinum Segmented Nanobattery. J. Am. Chem. Soc. 2011, 133, 20064-20067.

(9) Salem, A. K.; Searson, P. C.; Leong, K. W. Multifunctional Nanorods for Gene Delivery. Nat. Mater. 2003, 2, 668-671.

(10) Park, S.; Son, Y. J.; Leong, K. W.; Yoo, H. S. Therapeutic Nanorods with Metallic MultiSegments: Thermally Inducible Encapsulation of Doxorubicin for Anti-cancer Therapy. Nano Today 2012, 7, 76-84.

(11) Nicewarner-Peña, S. R.; Freeman, R. G.; Reiss, B. D.; He, L.; Peña, D. J.; Walton, I. D.; Cromer, R.; Keating, C. D.; Natan, M. J. Submicrometer Metallic Barcodes. Science 2001, 294, 137-141.

(12) Walton, I. D.; Norton, S. M.; Balasingham, A.; He, L.; Oviso, D. F.; Gupta, D.; Raju, P. A.; Natan, M. J.; Freeman, R. G. Particles for Multiplexed Analysis in Solution: Detection and Identification of Striped Metallic Particles Using Optical Microscopy. Anal. Chem. 2002, 74, 2240-2247.

(13) Al-Mawlawi, D.; Liu, C.; Moskovits, M. Nanowires Formed in Anodic Oxide Nanotemplates. J. Mater. Res. 1994, 9, 1014-1018.

(14) Hulteen, J. A General Template-based Method for the Preparation of Nanomaterials. J. Mater. Chem. 1997, 7, 1075-1087.

(15) Martin, B. R.; Dermody, D. J.; Reiss, B. D.; Fang, M.; Lyon, L. A.; Natan, M. J.; Mallouk, T. E. Orthogonal Self-Assembly on Colloidal Gold-Platinum Nanorods. Adv. Mater. 1999, 11, 1021-1025.

(16) Anderson, M. E.; Buck, M. R.; Sines, I. T.; Oyler, K. D.; Schaak, R. E. On-Wire Conversion Chemistry: Engineering Solid-State Complexity into Striped Metal Nanowires using Solution Chemistry Reactions. J. Am. Chem. Soc. 2008, 130, 1404214043.

(17) Leonard, B. M.; Anderson, M. E.; Oyler, K. D.; Phan, T.-H.; Schaak, R. E. Orthogonal Reactivity of Metal and Multimetal Nanostructures for Selective, Stepwise, and SpatiallyControlled Solid-State Modification. ACS Nano 2009, 3, 940-948. 
(18) Savas, T.; Schattenburg, M.; Carter, J.; Smith, H. I. Large-area Achromatic Interferometric Lithography for $100 \mathrm{~nm}$ Period Gratings and Grids. J. Vac. Sci. \& Tech. B 1996, 14, 4167-4170.

(19) Nikoobakht, B.; El-Sayed, M. A. Preparation and Growth Mechanism of Gold Nanorods (NRs) Using Seed-Mediated Growth Method. Chem. Mater. 2003, 15, 1957-1962.

(20) Gole, A.; Murphy, C. J. Seed-Mediated Synthesis of Gold Nanorods: Role of the Size and Nature of the Seed. Chem. Mater. 2004, 16, 3633-3640.

(21) Habas, S. E.; Lee, H.; Radmilovic, V.; Somorjai, G. A.; Yang, P. Shaping Binary Metal Nanocrystals through Epitaxial Seeded Growth. Nat. Mater. 2007, 6, 692-697.

(22) Lim, B.; Jiang, M.; Camargo, P. H.; Cho, E. C.; Tao, J.; Lu, X.; Zhu, Y.; Xia, Y. Pd-Pt Bimetallic Nanodendrites with High Activity for Oxygen Reduction. Science 2009, 324, 1302-1305.

(23) DeSantis, C. J.; Sue, A. C.; Bower, M. M.; Skrabalak, S. E. Seed-Mediated Co-reduction: A Versatile Route to Architecturally Controlled Bimetallic Nanostructures. ACS Nano 2012, 6, 2617-2628.

(24) Chen, S.; Jenkins, S. V.; Tao, J.; Zhu, Y.; Chen, J. Anisotropic Seeded Growth of Cu-M $(\mathrm{M}=\mathrm{Au}, \mathrm{Pt}$, or Pd) Bimetallic Nanorods with Tunable Optical and Catalytic Properties. $J$. Phys. Chem. C 2013, 117, 8924-8932.

(25) Pellegrino, T.; Fiore, A.; Carlino, E.; Giannini, C.; Cozzoli, P. D.; Ciccarella, G.; Respaud, M.; Palmirotta, L.; Cingolani, R.; Manna, L. Heterodimers Based on $\mathrm{CoPt}_{3}-\mathrm{Au}$ Nanocrystals with Tunable Domain Size. J. Am. Chem. Soc. 2006, 128, 6690-6698.

(26) Lim, B.; Kobayashi, H.; Yu, T.; Wang, J.; Kim, M. J.; Li, Z.-Y.; Rycenga, M.; Xia, Y. Synthesis of Pd-Au Bimetallic Nanocrystals via Controlled Overgrowth. J. Am. Chem. Soc. 2010, 132, 2506-2507.

(27) Zeng, J.; Zhu, C.; Tao, J.; Jin, M.; Zhang, H.; Li, Z. Y.; Zhu, Y.; Xia, Y. Controlling the Nucleation and Growth of Silver on Palladium Nanocubes by Manipulating the Reaction Kinetics. Angew. Chem. Int. Ed. 2012, 51, 2354-2358.

(28) Zhu, C.; Zeng, J.; Tao, J.; Johnson, M. C.; Schmidt-Krey, I.; Blubaugh, L.; Zhu, Y.; Gu, Z.; Xia, Y. Kinetically Controlled Overgrowth of Ag or Au on Pd Nanocrystal Seeds: From Hybrid Dimers to Nonconcentric and Concentric Bimetallic Nanocrystals. J. Am. Chem. Soc. 2012, 134, 15822-15831.

(29) Yang, Y.; Wang, W.; Li, X.; Chen, W.; Fan, N.; Zou, C.; Chen, X.; Xu, X.; Zhang, L.; Huang, S. Controlled Growth of Ag/Au Bimetallic Nanorods through Kinetics Control. Chem. Mater. 2012, 25, 34-41.

(30) Xie, S.; Peng, H.-C.; Lu, N.; Wang, J.; Kim, M. J.; Xie, Z.; Xia, Y. Confining the Nucleation and Overgrowth of Rh to the $\{111\}$ Facets of Pd Nanocrystal Seeds: The Roles of Capping Agent and Surface Diffusion. J. Am. Chem. Soc. 2013, 135, 1665816667.

(31) Chen, T.; Chen, G.; Xing, S.; Wu, T.; Chen, H. Scalable Routes to Janus $\mathrm{Au}-\mathrm{SiO}_{2}$ and Ternary $\mathrm{Ag}-\mathrm{Au}-\mathrm{SiO}_{2}$ Nanoparticles. Chem. Mater. 2010, 22, 3826-3828.

(32) Ohmori, M.; Matijević, E. Preparation and Properties of Uniform Coated Inorganic Colloidal Particles: 8. Silica on Iron. J. Colloid Interface Sci. 1993, 160, 288-292.

(33) Liz-Marzán, L. M.; Philipse, A. P. Synthesis and Optical Properties of Gold-Labeled Silica Particles. J. Colloid Interface Sci. 1995, 176, 459-466.

(34) Roca, M.; Haes, A. J. Silica-Void-Gold Nanoparticles: Temporally Stable SurfaceEnhanced Raman Scattering Substrates. J. Am. Chem. Soc. 2008, 130, 14273-14279. 
(35) Guerrero-Martínez, A.; Pérez-Juste, J.; Liz-Marzán, L. M. Recent Progress on Silica Coating of Nanoparticles and Related Nanomaterials. Adv. Mater. 2010, 22, 1182-1195.

(36) Johnson, C. L.; Snoeck, E.; Ezcurdia, M.; Rodriguez-Gonzalez, B.; Pastoriza-Santos, I.; Liz-Marzan, L. M.; Hytch, M. J. Effects of Elastic Anisotropy on Strain Distributions in Decahedral Gold Nanoparticles. Nat. Mater. 2008, 7, 120-124.

(37) Jungjohann, K. L.; Bliznakov, S.; Sutter, P. W.; Stach, E. A.; Sutter, E. A. In Situ Liquid Cell Electron Microscopy of the Solution Growth of Au-Pd Core-Shell Nanostructures. Nano Lett. 2013, 13, 2964-2970.

(38) Kibler, L. A.; Kleinert, M.; Randler, R.; Kolb, D. M. Initial Stages of Pd Deposition on Au(hkl) Part I: Pd on Au(111). Surf. Sci. 1999, 443, 19-30.

(39) Naohara, H.; Ye, S.; Uosaki, K. Electrochemical Layer-by-Layer Growth of Palladium on an $\mathrm{Au}(111)$ Electrode Surface: Evidence for Important Role of Adsorbed Pd Complex. The Journal of Physical Chemistry B 1998, 102, 4366-4373.

(40) Ding, Y.; Fan, F.; Tian, Z.; Wang, Z. L. Atomic Structure of Au-Pd Bimetallic Alloyed Nanoparticles. J. Am. Chem. Soc. 2010, 132, 12480-12486.

(41) Koel, B. E.; Sellidj, A.; Paffett, M. T. Ultrathin Films of Pd on Au(111): Evidence for Surface Alloy Formation. Phys. Rev. B 1992, 46, 7846-7856.

(42) Shibata, T.; Bunker, B. A.; Zhang, Z.; Meisel, D.; Vardeman, C. F.; Gezelter, J. D. SizeDependent Spontaneous Alloying of $\mathrm{Au}-\mathrm{Ag}$ Nanoparticles. J. Am. Chem. Soc. 2002, 124, 11989-11996.

(43) Langhammer, C.; Yuan, Z.; Zoric, I.; Kasemo, B. Plasmonic Properties of Supported Pt and Pd Nanostructures. Nano Lett. 2006, 6, 833-838.

(44) Draine, B. T.; Flatau, P. J. Discrete-Dipole Approximation for Scattering Calculations. J. Opt. Soc. Am. A 1994, 11, 1491-1499.

(45) Liao, H.-G.; Cui, L.; Whitelam, S.; Zheng, H. Real-time Imaging of $\mathrm{Pt}_{3} \mathrm{Fe}$ Nanorod Growth in Solution. Science 2012, 336, 1011-1014.

(46) Li, D.; Nielsen, M. H.; Lee, J. R.; Frandsen, C.; Banfield, J. F.; De Yoreo, J. J. Directionspecific Interactions Control Crystal Growth by Oriented Attachment. Science 2012, 336, 1014-1018.

(47) Waibel, H.-F.; Kleinert, M.; Kibler, L.; Kolb, D. Initial Stages of Pt Deposition on Au (111) and Au (100). Electrochim. Acta 2002, 47, 1461-1467.

(48) Sanchez, S. I.; Small, M. W.; Zuo, J.-m.; Nuzzo, R. G. Structural Characterization of $\mathrm{Pt}-\mathrm{Pd}$ and $\mathrm{Pd}-\mathrm{Pt}$ Core-Shell Nanoclusters at Atomic Resolution. J. Am. Chem. Soc. 2009, 131, 8683-8689.

(49) Koole, R.; van Schooneveld, M. M.; Hilhorst, J.; de Mello Donegá, C.; Hart, D. C. '.; van Blaaderen, A.; Vanmaekelbergh, D.; Meijerink, A. On the Incorporation Mechanism of Hydrophobic Quantum Dots in Silica Spheres by a Reverse Microemulsion Method. Chem. Mater. 2008, 20, 2503-2512.

(50) Park, S.-J.; Kim, Y.-J.; Park, S.-J. Size-Dependent Shape Evolution of Silica Nanoparticles into Hollow Structures. Langmuir 2008, 24, 12134-12137.

(51) Herves, P.; Pérez-Lorenzo, M.; Liz-Marzán, L. M.; Dzubiella, J.; Lu, Y.; Ballauff, M. Catalysis by Metallic Nanoparticles in Aqueous Solution: Model Reactions. Chem. Soc. Rev. 2012, 41, 5577-5587.

(52) Hung, L.-I.; Tsung, C.-K.; Huang, W.; Yang, P. Room-Temperature Formation of Hollow $\mathrm{Cu}_{2} \mathrm{O}$ Nanoparticles. Adv. Mater. 2010, 22, 1910-1914. 
(53) Motl, N. E.; Bondi, J. F.; Schaak, R. E. Synthesis of Colloidal Au-Cu 2 S Heterodimers via Chemically Triggered Phase Segregation of AuCu Nanoparticles. Chem. Mater. 2012, 24, 1552-1554. 


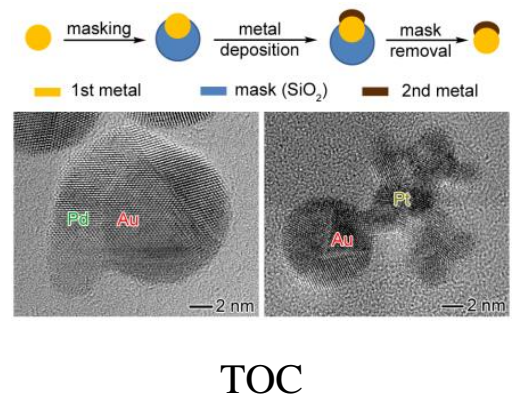

